\title{
Adult skeletal muscle stem cell migration is mediated by a blebbing/amoeboid mechanism
}

Article

Accepted Version

Otto, A., Collins-Hooper, H., Patel, A., Dash, P. R. and Patel, K. (2011) Adult skeletal muscle stem cell migration is mediated by a blebbing/amoeboid mechanism. Rejuvenation Research, 14 (3). pp. 249-260. ISSN 1549-1684 doi: https://doi.org/10.1089/rej.2010.1151 Available at https://centaur.reading.ac.uk/18088/

It is advisable to refer to the publisher's version if you intend to cite from the work. See Guidance on citing.

To link to this article DOI: http://dx.doi.org/10.1089/rej.2010.1151

Publisher: Mary Ann Liebert

All outputs in CentAUR are protected by Intellectual Property Rights law, including copyright law. Copyright and IPR is retained by the creators or other copyright holders. Terms and conditions for use of this material are defined in the End User Agreement.

www.reading.ac.uk/centaur 
Central Archive at the University of Reading

Reading's research outputs online 
Adult skeletal muscle stem cell migration is mediated by blebbing / amoeboid mechanism

Anthony Otto ${ }^{1}$, Henry Collins-Hooper ${ }^{1}$, Anand Patel, , Philip R Dash and Ketan Patel*

School of Biological Sciences, Hopkins Building, University of Reading, Whiteknights Campus, Reading, Berkshire, RG6 6UB, UK.

1- Joint-first authors

*Corresponding author:

Professor Ketan Patel

School Biological Sciences

Hopkins Building

University of Reading

Whiteknights

Reading

Berkshire, RG6 6UB

UK

Tel (0044) 01183788079

ketan.patel@ reading.ac.uk

Short title: Satellite cell bleb-based migration

Key words: Bleb, migration, satellite, amoeboid, stem, cell, skeletal, muscle. 


\section{Summary}

Adult skeletal muscle possesses a resident stem cell population called satellite cells which are responsible for tissue repair following damage. Satellite cell migration is crucial in promoting rapid tissue regeneration but is a poorly understood process. Furthermore, the mechanisms facilitating satellite cell movement have yet to be elucidated. Here the process of satellite cell migration has been investigated revealing that they undergo two distinct phases of movement; firstly under the basal lamina and then rapidly increasing their velocity when on the myofibre surface. Most significantly we show that satellite cells move using a highly dynamic blebbing based mechanism and not via lamellopodia mediated propulsion. We show that nitric oxide and non-canonical Wnt signalling pathways are necessary for regulating the formation of blebs and the migration of satellite cells. In summary, we propose that the formation of blebs and their necessity for satellite cell migration has significant implications in the future development of therapeutic regimes aimed at promoting skeletal muscle regeneration. 


\section{Introduction}

Adult mammalian skeletal muscle possesses a remarkable capacity for repair and regeneration of damaged tissue following injury or overwork. The repair and maintenance of this tissue is primarily carried out by a population of stem cells known as satellite cells that are located beneath the basal lamina that surrounds each myofibre [1]. Satellite cells are maintained in a metabolically quiescent state in undamaged muscle but are activated following muscle injury. The cells subsequently undergo cell division to form a pool of new myoblasts which then differentiate to form myotubes, replacing or supplementing existing muscle [2]. A much neglected feature of regeneration is the necessity for satellite cells to migrate to the lesion focus in order to carry out cellular tissue repair. Recent work has shown that matrix metalloproteinase which promote muscle cell migration and differentiation significantly increases the size of myoblast engraftment territory in a regenerating model [3], Furthermore the importance of this migration helps explain the failure, in both animal models and in humans, of numerous myoblast injection trials aimed at restoring muscle function [4-6]. In such studies, cells rarely moved more than $200 \mu \mathrm{m}$ from the point of injection and did not display the requisite whole muscle dispersal. Understanding the regulation of satellite cell migration is of fundamental importance in the development of new therapies for the treatment of muscular dystrophies and age-related impairment of muscle regeneration.

A few studies have suggested that activated satellite cells can migrate along the entire length of myofibres in vivo and are also capable of moving between fibres [7-8]. However, experimental proof for this notion in vivo is still lacking. Indeed there is much debate regarding the exact location of satellite cell migration with evidence for cell movement taking place both above and below the basal lamina (Chemotaxis in myogenesis (1996) Grounds MD and Davies MJ. Basic and Applied Myology 6 p 469-483). Migration has been traditionally explained by the force generated by structures called lamellipodia that form at the leading edge of the cells and the retraction of the cell at the rear. The propagation of the lamellipodia has been shown to be mediated by the continuous growth of filamentous actin through the process of 
monomer polymerisation at the front of the cell [9]. Most studies investigating muscle cell migration have been limited to using immortalized myoblast cell lines and support the notion that these cells move using lamellipodia [10-14]. A recent elegant study by Siegel et al [15] investigated satellite cell migration on isolated myofibres as opposed to cell lines and revealed a number of novel behavioural characteristics of satellite cells including the ability of cells to move as cohorts [15]. The use of isolated myofibres permits the observation of primary satellite cells moving on their native substrate in a near physiological context and provides an opportunity to experimentally investigate the mechanisms that regulate their migration through the use of pharmacological inhibitors, blocking antibodies or the addition of growth factors or chemokines [13].

At presents the signalling molecules that regulate satellite cell migration remain undefined. However clues to their identity come from the key players implicated in cell migration in other biological contexts. Nitric oxide, a potent short lived signalling molecule regulates cell migration in numerous biological systems including endothelial, epithelial and trophoblast cells [16-18]. NO is produced by a group of enzymes known as the nitric oxide synthases (NOS) which exist in three isoforms - neuronal NOS (nNOS), endothelial NOS (eNOS) and inducible NOS (iNOS). Recent work shows that NO plays an important role in the regulation of satellite cell function including their activation following injury [19]. The second is the Wnt signalling pathway. This family of highly conserved secreted proteins regulate almost every stage of animal development and tissue homeostasis. We have previously shown that members of the Wnt family have a ligand-specific effect on satellite cell proliferation based on the canonical signalling pathway involving $\beta$-catenin [20]. However specific Wnt ligand and receptor combinations activate an alternative signalling cascade called the planar cell polarity pathway (PCP) which is extensively deployed during cell movement both during normal development and disease progression [21-22]. 
Previous work has suggested that satellite cells are a heterogeneous population, with approximately $10 \%$ of cells never having expressed the myogenic regulatory factor Myf5, and that the Myf5 sub-population correspond to the self-renewing skeletal muscle stem cells [23]. Pertinent to this study Kuang et al., [23] suggest that the initial division of satellite cells takes place within the original satellite cell niche beneath the basal lamina of the myofibre, and that it is the plane of division (perpendicular to, or parallel to the basal lamina) within this niche that determines the fate of daughter cells; perpendicular divisions result in asymmetric divisions within the satellite cell population, and that these divisions occur predominantly in the Myf5 ${ }^{-}$population [23].

The study of Siegel et al [15] has demonstrated the power of coupling timelapse microscopy with the single fibre isolation technique. In this study we aimed to establish the mechanism that permits satellite cell migration. Here we report a number of novel properties of motile satellite cells including evidence for lamellipodia based migration immediately after single fibre isolation. However our most striking e observation is that satellite cells employ blebbing or amoeboid-based movement to move along the myofibres after emerging from the basal lamina. Blebbing based migration is a mechanism permitting rapid cell movement mediated by the extension of plasma membrane protrusions into the extra cellular matrix driven by local hydrodynamic changes in cytosolic pressure [24], also reviewed by Charras and Paluch [25]. Blebbing occurs shortly after the cells emerge onto the surface of the myofibres and our time lapse microscopy studies capture satellite cells extending protrusions approximately $7 \%$ of cell length over periods of 15 seconds before initiating their retraction. Furthermore we have determined that satellite cells migrate at very high velocities. We also show that entire clusters of satellite cells can move along the myofibre and that this movement also requires membrane blebbing. The formation of blebs and the migration of satellite cells were found to be dependent on both nitric oxide and non-canonical Wnt signalling, suggesting possible new regulatory mechanisms for bleb formation and novel therapeutic targets for muscle regeneration. Finally we show that intracellular inhibition of a key regulator of bleb formation causes satellite cell to form lamellipodia. 


\section{Results}

\section{Migration of Satellite cells}

Investigations carried out by ourselves and others have shown that satellite cells undergo a programme of molecular activation a few hours after single fibre isolation and emerge from their sub-laminar position at about 24 hours $[15,20,26]$. We found that after this time point, cells are able to move rapidly at approximately $50 \mu \mathrm{m} / \mathrm{h}$ ( $\mathrm{n}=33$ cells tracked). This rate of migration is faster than most other cells examined to date (Figure 1A.) However detailed examination of migration speeds following single fibre isolation showed distinct phases of migration speed. We found that soon after fibre isolation (0-18 hours) the satellite cells moved, but at a considerably lower speed $(27 \mu \mathrm{m} / \mathrm{h}, \mathrm{n}=33$ cells tracked $)$ than at approximately 24 hours where they rapidly accelerated. After approximately 36 hours migration rates of satellite cells decreased and by 54 hours movement decreased further (Figure 1B). After approximately 72 hours, cells had formed clusters of progeny on the surface of myofibres (Supplementary Figure 1F arrowheads). Examination of time lapse movies revealed interesting migratory patterns, some previously noted by Siegel et al [15]. Although movement was decreased following 54 hours of culture, there were frequent instances when whole clusters of cells could be observed migrating as a cohort along the surface of myofibres, and the clusters subsequently splitting into separate groups of cells (Supplementary figure 1I-). We also found instances of satellite cells moving from one fibre to another (Supplementary figure 1L-N). At other times, individual satellite cells would migrate towards each other and then appear to make contact followed by them subsequently moving as a pair (Supplementary figure 1O-Q).

\section{Laminin remodelling prior to satellite cell emergence}

Previous work had reported that satellite cells emerged from their sub-laminar position after 24 hours [13] and yet we had detected migration prior to this event (Figure $1 \mathrm{~B}$ and supplementary movie $1 \mathrm{~A}$ ). We therefore carried out a detailed analysis of the emergence of satellite cells from under the basal lamina. 
Immediately after fibre isolation we are able to detect the basal lamina over the Pax7 expressing cell (Figure 1C). Importantly there was a uniform layer of laminin over the satellite cell and the myofibre. Subsequently, we found that the distribution of laminin changed to become concentrated around and above the satellite cell- a phase lasting approximately 18 hours (Figure 1D-G, red arrowheads and 1J dorsal view). From 18 hours onwards holes were noted in the concentrated basal lamina (Figure 1H-red arrowhead). Satellite cells appear to emerge from these holes in the basal lamina (Figure $1 \mathrm{~K}$ green arrow head). Emergence of the satellite cell often left a scarred basal lamina. By 24 hours, most of the satellite cells that had emerged had moved from their original resting place and were located over intact basal lamina (Figure 1I). Time lapse microscopy was used to examine satellite cell migration before emerging from the basal lamina. During this period, satellite cells displayed an elongated morphology and formed lamellipodia like projections ahead of the cell (Supplementary movie 1A)

\section{Migration characteristics of Satellite cells}

Satellite cells showed a remarkable ability to change direction and speed. We quantified movement by measuring the linear migration and found that straight-line movement was a rare event (see Figure 4B). One key observation was that satellite cells stopped moving during division (Supplementary figure 1B). However after division daughter cells showed one of three types of behaviour. One or both cells did not move, or both daughter cells moved together or as most commonly found the daughter cells moved independently of each other (as shown in Supplementary figure 1). Our time lapse movies also revealed an interesting feature about cell division. We found that most cells divided at right angles to the muscle fibre so that for a brief period of time one cell appeared to be on top of the other (Supplementary figure $1 \mathrm{C}$ and $\mathrm{G})$.

Blebbing of Satellite cells during migration 
In order to identify which mechanism regulated satellite cell migration we generated electron micrographs at differing time points after fibre isolation. SEM images clearly showed the emergence of satellite cells from a hole in the basal lamina (Figure 2A). Interestingly, at these time points, the surface of the cells appeared to be smooth. This morphology is greatly altered following emergence of the satellite cells from the basal lamina, when satellite cells began to display membrane blebs (Figure 2B). The distribution of the blebs would be polarised to one end of the cell. Blebs were still prominent following 48 hours and 72 hours of culture (Figure 2C and D). Furthermore blebbing was solely confined to the supra-basally positioned satellite cells and did not occur on the myofibre. We measured bleb size during the three phases of migration after emergence from under the basal lamina and found that bleb size remained fairly constant $(0.69 \mu \mathrm{m}$ at $24 \mathrm{~h}, 0.67 \mu \mathrm{m}$ at $48 \mathrm{~h}$ and $0.85 \mu \mathrm{m}$ at $72 \mathrm{~h})$. These extensions are of significant length and represent protrusions of approximately $7 \%$ of overall satellite cell size.

\section{Highly dynamic nature of Satellite cell blebbing}

In order to confirm that blebbing is the main mechanism by which satellite cells move, and that our SEM images were not the result of satellite cells undergoing apoptosis, we carried out two experiments. Firstly, myofibres were cultured in the presence of a caspase inhibitor (Z-VAD-FMK), for 48 hours - a time point when satellite cell migration was at its most rapid, and myofibres were processed for SEM. SEM images revealed that satellite cells cultured in the presence of caspase inhibitor continued to bleb profusely (Figure 2E). Time lapse phase contrast microscopy was used to visualise single satellite cells moving along the surface of myofibres, and to determine whether the blebbing on the satellite cells was a dynamic process. These movies, taken 30-48 hrs following myofibre isolation over a period of 20-60 minutes, showed in all cases ( $\mathrm{n}=20$ tracked cells) that cells formed bleb-like protrusions and did not produce obvious lamellipodia (see Supplementary movie 1B).There was a significant difference in the morphology displayed by satellite cells that were positioned either on top of the fibre or under the basal lamina. Satellite cells positioned on the surface of myofibres were spherical in contrasted to the elongated shape when localised under the basal lamina. One of the fascinating features of blebbing is the 
highly dynamic nature of plasma membrane protrusion and retraction [24]. We found that on the surface of satellite cells, blebs were rapidly extended (15 seconds) and retracted at similarly high speeds (30-45 seconds) with the whole process taking place in approximately 60 seconds (Figure 5B and Supplementary movie 1). Blebbing occurred over the entire surface of the satellite cells but was more pronounced at the leading region (Supplementary movie 1B)

\section{Regulation of Satellite Cell migration}

Here we firstly aimed to establish a role for nitric oxide as a mediator of satellite cell migration. Nitric oxide is produced by a family of enzymes called nitric oxide synthases (NOS). We found a dynamic expression of endothelial NOS (eNOS) and inducible NOS (iNOS) in satellite cells. eNOS was not detected in quiescent satellite cells but was present following activation. Furthermore its expression was found in both the cytoplasm and at the cell membrane. Moreover, it was differentially expressed at high and low levels in cells of the same cluster or progeny (compare red and blue arrowheads in Figure 3B). Additionally its expression at the membrane was, at times, polarised to one end of the cell (Figure 3B red arrowheads).

In contrast, iNOS expression localised to the nuclei of the satellite cells and to a lesser degree that of the myonuclei (Figure 3A).

The role of NO and Wnt signalling in satellite cell migration

We investigated whether the NO and Wnt signalling pathway were able to regulate satellite cell migration. We took advantage of inhibitors that prevent signalling mediated by these molecules. LNAME acts a competitive inhibitor of all forms of NOS and prevents the synthesis of nitric oxide. The JNK/SAPK, c-Jun N-terminal kinases/stress-activated protein kinase (c-jnk) has been shown to regulate cell migration [27] and is a component of the non-canonical Wnt signalling pathway and can be inhibited using SP600125 [28]. 
Firstly we incubated isolated muscle fibres at differing phases of migration with either L-NAME or SP600125 and measured migration parameters of tracked satellite cells ( $\mathrm{n}=7$ cells tracked for L-NAME and 11 cells tracked for SP600125 respectively). L-NAME attenuated the rapid cell movement displayed by untreated cells (Figure 4A). In contrast to control cells which displayed an increased change in migration speeds over time, L-NAME treatment rendered the cells slow moving throughout the course of observation. Treatment with SP600125 had quite a different outcome on satellite cell migration. We found that during the first 18 hours there was no difference in the migration rates compared to untreated cells (Figure 4A). However after 18 hours, SP600125 brought approximately a 30\% average reduction in migration speed in all cells, which carried on reducing over time (Figure 4A).

Next we determined whether the NO or PCP signalling cascades regulated directionality of migration by calculating the straight line migration characteristics of satellite cells treated with either L-NAME or SP600125. Our results showed that although both inhibitors decreased the velocity of migration, they did not alter the directionality (Figure 4B).

\section{The effect of inhibiting PCP and NO signalling on satellite bleb formation}

We next examined whether the PCP and NP inhibitor compounds altered bleb morphology. Inhibition of the nitric oxide pathway with L-NAME led to very few blebs developing on satellite cells at all time points examined (Figure 5C). In contrast to untreated cells at 24 hours that showed polarised blebbing events. At 48 hours when control cells were covered in blebs, the number on L-NAME treated fibres was again considerably lower. Furthermore the morphology of blebs in L-NAME treated samples was altered compared to untreated fibres; L-NAME caused blebs to be much broader. A similar bleb profile was seen at 72 hours in the presence of L-NAME (Figure 5C).

Treatment with SP600125 also brought about a dramatic alteration in bleb number and morphology. At all time points there were fewer blebs in SP600125 treated samples. This was particularly evident at 72 
hours when the bleb number was severely decreased and those blebs that were present were large and flat compared to those on untreated cells (Figure 5C).

We quantified the number of blebs that formed at differing time points in the presence of the two inhibitors on satellite cells in SEM images. In untreated cells, the number of blebs had increased approximately 4-fold by the 72 hour experimental period (Figure 5A, n=3, 21 and 14 cells analyzed at 24, 48 and 72 hrs respectively). In contrast, treatment with either L-NAME or SP600125 resulted in fewer blebs at any comparable time point. Furthermore there was a small increase at 48 hours compared to 24 hours but that increase was only transient and at 72 hours bleb number was similar to that at 24 hours (Figure 5A, n=3, 10 and 15 cells for SP600125- and 4.10 and 33 cells for L-NAME-treated cells at 24, 48 and 72 hours respectively).

In order to confirm these effects of L-NAME and SP600125 on bleb formation, high power timelapse movies of single cells treated with both L-NAME and SP600125 were obtained at 48 hours following myofibre isolation. A key observation over short periods was that the satellite cells treated with either LNAME or SP600125 did not move but nevertheless did undergo blebbing. L-NAME treated fibres revealed two features. Firstly, the number of blebs that were seen developing on the cells was reduced and secondly that they were larger when they did form (albeit not as large as those observed in SP600125 treated cells). Examination with high power time lapse phase contrast microscopy of SP600125 treated cells revealed that cellular blebs were increased in size dramatically, reduced in number and the cell displayed disorganised erratic small movements from side to side as opposed to small organised bleb formation observed on control cells (see Supplementary movie 1C and D respectively).

\section{Actin cytoskeletal remodelling and ROCK-1activity are required for satellite cell blebbing}

We explored the role of Actin cytoskeletal remodelling in bleb formation by firstly monitoring blebbing in satellite cells at 48 hours following fibre isolation. Then we watched each cell following the 
introduction of Cytochalasin-D, an inhibitor of actin polymerisation [29]. We found that in all cases examined in the presence of Cytochalasin-D, cells extended their blebs, however the blebs then failed to retract (Figure 6A-C).

In order to investigate the role of ROCK in satellite cell bleb formation, we incubated myofibres in the presence of the ROCK inhibitor Y-27362 at 3 separate time points; T0-24, T24-48 and T48-72 hours respectively and carried out SEM analysis to establish satellite cell membrane morphology. Our data show that in the presence of Y-27362, all satellite cells viewed under SEM at all time points analysed adopted a smooth surface and produced lamellipodia like structures (Figure 6D, E and F and Supplementary movie 1E). We measured the speed of satellite cell migration following Y-27362 treatment between T48 and T56 hours following myofibre isolation and found that velocity was reduced from $41.3 \mu \mathrm{m} / \mathrm{h}$ in control conditions to $17.2 \mu \mathrm{m} / \mathrm{h}$ in the presence of Y-27632 (see Supplementary movie 1E).

\section{Inhibition of $\mathrm{NO}$ and PCP on satellite cell development}

We next determined whether the decrease in migration rates seen following the introduction of L-NAME or SP600125 was due to an acceleration in the differentiation programme. To this end we cultured fibres in either inhibitor and profiled the progeny after 72 hours of culture for myogenin as a marker for differentiation and for Pax7 as a marker of the self renewing myogenic stem cell population. We found that introduction of either inhibitor reduced the level of differentiation (Figure 5D). In conclusion, we can state that the decrease in migratory rates and the loss of blebbing is not due to the ability of the inhibitors to drive premature differentiation.

NO and PCP signalling and migration of cultured myoblasts.

We finally examined whether NO or PCP cascades regulated migration of muscle cells in-vitro. To this end we examined the effects of the inhibitors on the movement of $\mathrm{C} 2 \mathrm{C} 12$ cells. This myoblast cell line 
was grown under proliferation-maintaining conditions at all times during the experiment and introduction of either L-NAME or SP600125 resulted in a significant decrease in the rate of migration (Supplementary Figure 2). One difference was that SP600125 in these cultured cells caused a greater reduction in migration compared to L-NAME. We also found that simultaneous addition of both compounds resulted in a greater degree of inhibition of migration than either compound on its own (See Supplementary movie $1 \mathrm{~F})$. 


\section{Discussion}

Plasma membrane blebs are dynamic, cytoskeleton regulated structures that have long been known to be involved in apoptosis, but their role in cell migration has been overlooked until recently. The ability of cells to use membrane blebs for their motility has lately become an area of intense interest following the observation that primordial germ cells in vivo migrate exclusively through a bleb-mediated mechanism [30]. Furthermore new studies have shown that tumour cells can adopt a bleb-driven, amoeboid type of movement through extracellular matrix [31-32]. This bleb-driven migration produces faster migration speeds than the well studied lamellipodia-driven process. In this study we provide the first evidence that resident muscle stem cells use this newly discovered mechanism for migration along myofibres.

An essential feature in the regeneration process is thought to be the requirement of satellite cells to migrate to the point of lesion [7-8], although most studies on satellite cell migration have been performed using myoblast cell lines in culture [10-14]. Recently, primary satellite cells moving on the surface of myofibres have been observed using timelapse microscopy and a number of unexpected properties were reported in an important study by Siegel et al [15]. In particular, satellite cells on fibres showed a rapid migration compared to myoblast cell lines. Furthermore, numerous differences were noted between satellite cells and cultured muscle cell lines. The most significant finding of our study is that it represents the first report of satellite cells moving on their in-vivo substrate in an amoeboid or bleb-dependent mechanism, which is independent of lamellipodia formation. In addition to this startling finding we also confirm the rapid migration of satellite cells along myofibres and establish that the cell movement is more rapid than any other cell line tested, including the epithelial MCF10A cell line, which is widely used in migration studies due to its motile properties [33-34]. Previous studies have suggested that satellite cell asymmetric division occurs in, and is regulated by the stem cell niche environment, and occurs beneath the basal lamina of the myofibre [23]. However, our immunofluorescence, confocal, SEM and timelapse analysis all suggest that the initial division of all observed satellite cells occurs away from the original 
niche and only once the cells have emerged from beneath the basal lamina. Moreover, these experiments revealed that satellite cells were always stationary prior to and during division, and images captured during these phases using SEM showed no membrane blebbing, again evidence for blebbing being involved in migration. Interestingly, we have observed that the initial satellite cell division always occurs in the direction away from the myofibre surface, suggesting indeed that if asymmetric divisions are occurring, they may be regulated by an interaction with the outside, and not the inside, of the basal lamina. Further studies addressing this important issue are currently underway.

We also report that satellite cell migration starts even before the cells emerge from the basal lamina, with cells moving beneath the basal lamina at a relatively slow speed $(27 \mu \mathrm{m} / \mathrm{h})$, before stopping, emerging onto the surface and then start moving again at a much higher speed $(50 \mu \mathrm{m} / \mathrm{h})$. Given that cells emerge onto the surface of the myofibres with smooth membranes showing no sign of blebbing and that there are significant differences in the speed of migration on the surface compared to beneath the basal lamina it is possible that the satellite cells employ different mechanisms of migration depending on the environmental context in which they find themselves. Our time lapse movies support this notion by capturing images of elongated cells displaying lamellipodia like extensions in the direction of cell movement. The movement of satellite cells beneath the basal lamina may be a response to activation of the cells that leads them to search out potential exit locations to emerge onto the surface of the fibre, suggesting that their quiescent niche may not be optimum to enable exit from the basal lamina.

Perhaps the most startling behaviour observed is the formation of blebs on the surface of actively migrating satellite cells. In the early stages after satellite cells emerge onto the fibre scanning electron microscopy reveals that the emerging satellite cells have smooth surfaces. In some cases the beginnings of polarised blebbing can be observed shortly after emergence while at time points in which the cells are most motile (24-48 hours) all of the satellite cells, with the exception of a few that had recently undergone cell division, showed very substantial membrane blebbing. High magnification timelapse imaging of 
satellite cells moving along fibres also revealed substantial membrane blebbing occurring in all motile cells. Blebbing motility, also sometimes known as amoeboid motility, has recently begun to attract significant interest as an alternative mechanism of cell movement to that based on the more widely studied lamellipodia based motility. A number of recent studies suggest that bleb-based cell migration may be more common than is currently appreciated and may be a particular feature of cell migration in vivo (for reviews see Charras and Paluch [25] and Fackler and Grosse [35]. Live studies in zebrafish embryos have shown that primordial germ cells use blebs to migrate [30] while studies in tumour cells migrating through extracellular matrix (ECM) have revealed that tumour cells can employ a blebbing based mechanism of motility, distinct from lamellipodia based migration, to migrate through the ECM in a mechanism that does not require total matrix degradation [32, 36-37]. We now report the first instance of bleb-directed cell migration occurring in adult mammalian cells on their native substrate performing a physiological role.

Bleb formation appears to follow a well defined life cycle that can be divided into three phases initiation, expansion and retraction. The total life cycle of a bleb has been documented to last for 1-2 minutes and produces membrane blebs that expand up to $2 \mu \mathrm{m}$ in size. The dynamics of blebs formation in satellite cells appears to conform to these phases and have a life cycle of around 1 minute and can grow to a size of $0.7 \mu \mathrm{m}$. In addition to observing blebbing occurring in migrating satellite cells we have also observed significant blebbing occurring in entire clusters of cells. Since these clusters are also capable of rapid migration along the myofibre, it is interesting to note that they appear to employ the same mechanism of migration as the individual cells.

It has been proposed that blebbing based motility may provide a number of advantages to cells moving through complex 3D environments such as those found in muscle. In these environments lamellipodia may have no obvious surface to follow, bleb protrusion can occur in any direction making rapid changes in course possible and blebs may provide a simple means of adaptation to the shape and structure of the 
local environment. In addition bleb expansion is faster than lamellipodial protrusion suggesting that this may allow more rapid migration of cells. In tumour cells it has been proposed that the acquisition of amoeboid or blebbing based motility is associated with a greater ability to move and to colonize distant sites in vivo [38]. These observations are particularly important in light of our results which show that satellite cells can move along myofibres significantly faster than almost every cell line tested is capable of moving across a 2D surface using lamellipodia. In the context of muscle regeneration bleb based migration of satellite cells may provide a mechanism for rapid repair of damaged sites on muscle fibres. We suggest that a number of behavioural characteristics of satellite cells are governed by their local environment. An elegant study by Gilbert and colleagues has recently shown that substratum mechanical properties regulate the self-renewal capacity of satellite cells (NEW REF PMID: 20647425). We suggest that the mechanism used by satellite cells for migration is also dictated by the substratum. We have examined satellite cells maintained on myofibres in conditions that support proliferation and shown that they form blebs. In contrasts, others have taken satellite cells away from the myofibre, cultured them in differentiation medium and shown that they form lamellipodia (NEW REF PMID: 20736301).

Nitric oxide (NO) is a gaseous signalling molecule which regulates diverse physiological and cellular processes. It promotes cell migration in numerous cell types. Treatment of isolated myofibres with the general NOS inhibitor L-NAME led to significantly reduced migration and to impaired bleb formation. Blebbing in L-NAME treated cells was markedly reduced with fewer, smaller blebs occurring, suggesting that NO may play a role in the regulation of bleb formation. The role of NO in cell migration has exclusively been studied in cells employing lamellipodial motility rather than bleb based motility and many of the molecular targets for NO have been identified in these cells including the integrins $\alpha \mathrm{V} \beta 3$ [39] and $\beta 1$ [40]. It is possible that these NO-regulated proteins may also be involved in regulating bleb formation or it may be that there are further targets to be identified that are specific to blebbing based motility. 
We have previously shown that Wnt signalling plays a role in satellite cell proliferation dynamics [20] and wanted to determine the role non-canonical Wnt signalling might play in regulating satellite cell migration. The JNK/SAPK, c-Jun N-terminal kinases/stress-activated protein kinase has been shown to regulate cell migration [27] and is a component of the non-canonical Wnt signalling pathway. Using a selective inhibitor of JNK, a key component of the non-conical Wnt pathway we have shown that, like NO, it plays an important role in regulating both bleb formation and satellite cell migration. Little is known about the regulation of bleb formation in migrating cells and while much work is needed to fully elucidate these regulatory mechanisms we have established a role for both NO and non-canonical Wnt signalling in this process. One important feature of our studies was that although both NO and the PCP pathway regulated the velocity of migration, neither had any impact on directionality of movement. Comparisons are invited between our findings and those of Siegel and colleagues [15]. In the latter study, the influence of guidance molecules was examined on migration velocity. They found that Hepatocyte Growth Factor (HGF) altered migration trajectory but not the velocity. Assimilating the finding of Siegel et al., [15] and those in the present study it appears that the mechanism regulating cell migration and guidance are independent processes.

The observation of these behaviours of satellite cells, particularly their reliance on bleb formation for migration is fundamental to developing therapies for muscular dystrophies or ageing related muscle weakness, as bleb based motility uses completely different pathways and regulatory mechanisms to lamellipodial dependent migration. Studies using the myoblast cell line $\mathrm{C} 2 \mathrm{C} 12$ for example suggested that N-WASP and WAVE2, activated downstream of PI3-kinase, play an important role in satellite cell migration [12]. However, these cells move in a lamellipodial dependent mechanism and as such are a poor model for studying the regulation of migration in satellite cells. Our analysis of $\mathrm{C} 2 \mathrm{C} 12$ migration demonstrates that L-NAME has less of an effect on these myoblast cells whereas the PCP pathway plays a greater role in their movement, the opposite of our findings in primary adult satellite cells. These data support the hypothesis that the balance of pathways regulating bleb-based and lamellipodia-based 
migration is different and suggests separate mechanisms are in place. We show that inhibiting a key regulator of amoeboid movement causes cells to no longer display blebs but instead from lamellipodia. This work supports the hypothesis that blebbing and lamellipodia based mechanisms acts in an antagonistic manner [41] .

Blebbing motility represents an important and exciting new avenue of research in the field of cell migration especially of satellites cells yet remains poorly studied compared to lamellipodial motility. This may be because its role in cell migration in vivo is under-appreciated, possibly due to the technical difficulty of studying this type of migration as it is only apparent in complex 3D matrices or living tissues. 


\section{Materials and Methods}

\section{Single myofibre culture}

Myofibres were isolated from the extensor digitorum longus (EDL) muscle of 4-month-old female Swiss mice as described previously [20]. Briefly, an undamaged EDL muscle was dissected with both tendons intact, and the singles fibres liberated through digestion with $0.1 \%$ type I Collagenase in DMEM at $37^{\circ} \mathrm{C}$ 5\% $\mathrm{CO}_{2}$. Using tapered glass pipettes, single fibres were plated out in floating culture wells containing DMEM supplemented with $10 \%$ horse serum and $0.5 \%$ chick embryo extract. When required, small molecule inhibitors $5 \mathrm{mM}$ L-NAME, or $25 \mu \mathrm{M}$ SP600125, were added to myofibre culture media from the point of culture initiation. $1 \mu \mathrm{M}$ Cytochalasin-D was added following $48 \mathrm{hrs}$ of culture in standard conditions. $10 \mu \mathrm{g} / \mathrm{ml}$ ROCK-1 inhibitor Y-27632 was added following 0, 24 or 48 hours of culture and 20 $\mu \mathrm{M}$ Caspase inhibitor Z-VAD-FMK was added following $24 \mathrm{hrs}$ of culture respectively.

\section{Timelapse Microscopy}

Myofibre cultures and $\mathrm{C} 2 \mathrm{C} 12$ myoblast cells were monitored using two separate phase contrast microscope systems that harbour controlled-environment chambers maintained at $37^{\circ} \mathrm{C}, 5 \% \mathrm{CO}_{2}$. Timelapse video was taken at rate of 1 frame every 15 minutes over 72 hrs for satellite cells using a 10X objective, a previously well studied time course of satellite cell progression following skeletal muscle injury [20, 26], or for $24 \mathrm{hrs}$ for $\mathrm{C} 2 \mathrm{C} 12$ cells. In separate analysis, high power timelapse microscopy using a 40X objective was carried out, enabling the visualisation of cell surface blebbing on single satellite cells. For such analysis, cells were cultured for $48 \mathrm{hrs}$ then selected and subsequently tracked at a rate of 1 frame per 20 seconds. 


\section{Immunocytochemistry}

Immunocytochemistry of satellite cells on single myofibres was carried out as described previously [20]. Briefly, myofibres were fixed gently in 2\% Paraformaldehyde in PBS (PFA) for 10 minutes and washed 3 times in PBS. Myofibres were permeabilised in a solution of $20 \mathrm{mM}$ Hepes, $300 \mathrm{mM}$ sucrose, $50 \mathrm{mM}$ $\mathrm{NaCl}, 3 \mathrm{mM} \mathrm{MgCl} l_{2}$ and $0.5 \%$ Triton-X100 $(\mathrm{pH} 7)$ at $4^{\circ} \mathrm{C}$ for 15 minutes and incubated in blocking wash buffer (5\% newborn calf serum in PBS containing $0.01 \%$ triton X-100) for 30 minutes prior to antibody incubation. Antibodies were diluted and pre-blocked in wash buffer for 30 minutes prior to addition to the myofibres. Primary antibodies used were: Polyclonal rabbit anti-Laminin (Sigma L-9393 1:200), monoclonal mouse anti-Pax7 (Developmental Studies Hybridoma Bank (DSHB) 1:1), polyclonal rabbitanti-myogenin (Santa Cruz biotechnology 1:200), polyclonal rabbit anti-iNOS (Santa Cruz Biotechnology 1:200) and polyclonal rabbit anti-eNOS (Santa Cruz Biotechnology 1:200). All primary antibodies were incubated over night at $4^{\circ} \mathrm{C}$. Primary antibodies were visualised using the following secondary antibodies: Alexa Fluor goat anti-mouse 488 (Molecular probes A11029) and Alexa Fluor goat anti-rabbit 633 (Molecular probes A21071). Secondary antibodies were used at 1:200 and incubated at room temperature for 45 minutes. All myofibres were mounted in Fluorescent mounting medium (DAKO) containing $7.5 \mathrm{mg} / \mathrm{ml}$ DAPI for nuclear visualisation.

\section{Fluorescence and confocal microscopy}

Mounted myofibres were analysed using a Zeiss Axioscope fluorescent microscope and images captured using an Axiocam digital camera system and Axiovision image analysis software (version 4.7). For confocal analysis, myofibres were analysed using a Leica DMIRE2 confocal microscope. Z-stack images were taken every $0.5 \mu \mathrm{M}$ and 3D reconstructions were performed using Leica confocal analysis software. 
Single myofibres were fixed following 24,48 , and $72 \mathrm{hrs}$ in standard culture conditions in $2 \%$ PFA for 10 minutes. Myofibres were dehydrated through 30, 50, 70, 80, 90, 100 and 100\% Ethanol solutions (15 minutes for each step) and transferred to a critical point drier (Balzers CPD 030 - using liquid carbon dioxide). Dried myofibres were then carefully transferred to SEM chucks using micro forceps under a light microscope. Myofibres were then gold-coated using an Edwards S150B sputter-coater. Coated myofibres were then analysed under a FEI 600F scanning electron microscope using the accompanying analysis software for image collection.

\section{C2C12 cell culture}

C2C12 cells were cultured in DMEM containing Glutamax and pyruvate supplemented with $1 \%$ penicillin/streptomycin and 10\% foetal calf serum. For migration analysis, cells were plated out in 4 well plates, supplemented with the appropriate inhibitors (L-NAME $(5 \mathrm{mM})$ and/or SP600125 $(25 \mu \mathrm{M})$ ) and grown until confluent. Plates were then taken for time lapse microscopy.

Image and movie analysis

All image analysis was carried out using freeware package ImageJ (version 1.4.3). Single C2C12 and satellite cells were individually manually tracked using the plugin MTrackJ. Bleb dynamics were quantified using ImageJ. Bleb number was quantified manually on SEM images of cells using Adobe Photoshop version CS2, only SEM images of whole cells were used for this quantification. Satellite cell differentiation profiles following differing culture conditions were manually assessed using quantification of live images through the Zeiss axioscope and axiovision digital camera system. 


\section{Acknowledgements}

We would like to thank the University of Reading, the BBSRC ( $\mathrm{KP}$ and $\mathrm{HCH})$ and the British Heart

Foundation (FS/08/056 - PD and AP) for generous funding to allow the completion of this study, and Dr

Chris Stain for assistance with SEM analysis. 


\section{References}

1. Collins, C.A., I. Olsen, P.S. Zammit, L. Heslop, A. Petrie, T.A. Partridge, and J.E. Morgan, Stem cell function, self-renewal, and behavioral heterogeneity of cells from the adult muscle satellite cell niche. Cell, 2005. 122(2): p. 289-301.

2. Zammit, P.S., L. Heslop, V. Hudon, J.D. Rosenblatt, S. Tajbakhsh, M.E. Buckingham, J.R. Beauchamp, and T.A. Partridge, Kinetics of myoblast proliferation show that resident satellite cells are competent to fully regenerate skeletal muscle fibers. Exp Cell Res, 2002. 281(1): p. 3949.

3. Wang, W., H. Pan, K. Murray, B.S. Jefferson, and Y. Li, Matrix metalloproteinase-1 promotes muscle cell migration and differentiation. Am J Pathol, 2009. 174(2): p. 541-9.

4. Peault, B., M. Rudnicki, Y. Torrente, G. Cossu, J.P. Tremblay, T. Partridge, E. Gussoni, L.M. Kunkel, and J. Huard, Stem and progenitor cells in skeletal muscle development, maintenance, and therapy. Mol Ther, 2007. 15(5): p. 867-77.

5. Lafreniere, J.F., P. Mills, J.P. Tremblay, and E. El Fahime, Growth factors improve the in vivo migration of human skeletal myoblasts by modulating their endogenous proteolytic activity. Transplantation, 2004. 77(11): p. 1741-7.

6. Satoh, A., J. Huard, C. Labrecque, and J.P. Tremblay, Use of fluorescent latex microspheres (FLMs) to follow the fate of transplanted myoblasts. J Histochem Cytochem, 1993. 41(10): p. 1579-82.

7. Phillips, G.D., J.R. Hoffman, and D.R. Knighton, Migration of myogenic cells in the rat extensor digitorum longus muscle studied with a split autograft model. Cell Tissue Res, 1990. 262(1): p. 81-8.

8. Schultz, E., D.L. Jaryszak, and C.R. Valliere, Response of satellite cells to focal skeletal muscle injury. Muscle Nerve, 1985. 8(3): p. 217-22.

9. Petrie, R.J., A.D. Doyle, and K.M. Yamada, Random versus directionally persistent cell migration. Nat Rev Mol Cell Biol, 2009. 10(8): p. 538-49.

10. Bae, G.U., U. Gaio, Y.J. Yang, H.J. Lee, J.S. Kang, and R.S. Krauss, Regulation of myoblast motility and fusion by the CXCR4-associated sialomucin, CD164. J Biol Chem, 2008. 283(13): p. 8301-9.

11. Dedieu, S., S. Poussard, G. Mazeres, F. Grise, E. Dargelos, P. Cottin, and J.J. Brustis, Myoblast migration is regulated by calpain through its involvement in cell attachment and cytoskeletal organization. Exp Cell Res, 2004. 292(1): p. 187-200.

12. Kawamura, K., K. Takano, S. Suetsugu, S. Kurisu, D. Yamazaki, H. Miki, T. Takenawa, and T. Endo, N-WASP and WAVE2 acting downstream of phosphatidylinositol 3-kinase are required for myogenic cell migration induced by hepatocyte growth factor. J Biol Chem, 2004. 279(52): p. 54862-71.

13. Nishimura, T., K. Nakamura, Y. Kishioka, Y. Kato-Mori, J. Wakamatsu, and A. Hattori, Inhibition of matrix metalloproteinases suppresses the migration of skeletal muscle cells. J Muscle Res Cell Motil, 2008. 29(1): p. 37-44.

14. Odemis, V., K. Boosmann, M.T. Dieterlen, and J. Engele, The chemokine SDF1 controls multiple steps of myogenesis through atypical PKCzeta. J Cell Sci, 2007. 120(Pt 22): p. 4050-9.

15. Siegel, A.L., K. Atchison, K.E. Fisher, G.E. Davis, and D.D. Cornelison, 3D timelapse analysis of muscle satellite cell motility. Stem Cells, 2009. 27(10): p. 2527-38.

16. Cartwright, J.E., W.K. Tse, and G.S. Whitley, Hepatocyte growth factor induced human trophoblast motility involves phosphatidylinositol-3-kinase, mitogen-activated protein kinase, and inducible nitric oxide synthase. Exp Cell Res, 2002. 279(2): p. 219-26. 
17. Kawasaki, K., R.S. Smith, Jr., C.M. Hsieh, J. Sun, J. Chao, and J.K. Liao, Activation of the phosphatidylinositol 3-kinase/protein kinase Akt pathway mediates nitric oxide-induced endothelial cell migration and angiogenesis. Mol Cell Biol, 2003. 23(16): p. 5726-37.

18. Noiri, E., T. Peresleni, N. Srivastava, P. Weber, W.F. Bahou, N. Peunova, and M.S. Goligorsky, Nitric oxide is necessary for a switch from stationary to locomoting phenotype in epithelial cells. Am J Physiol, 1996. 270(3 Pt 1): p. C794-802.

19. Anderson, J.E., A role for nitric oxide in muscle repair: nitric oxide-mediated activation of muscle satellite cells. Mol Biol Cell, 2000. 11(5): p. 1859-74.

20. Otto, A., C. Schmidt, G. Luke, S. Allen, P. Valasek, F. Muntoni, D. Lawrence-Watt, and K. Patel, Canonical Wnt signalling induces satellite-cell proliferation during adult skeletal muscle regeneration. J Cell Sci, 2008. 121(Pt 17): p. 2939-50.

21. Etienne-Manneville, S., APC in cell migration. Adv Exp Med Biol, 2009. 656: p. 30-40.

22. Jessen, J.R., Noncanonical Wnt signaling in tumor progression and metastasis. Zebrafish, 2009. 6(1): p. 21-8.

23. Kuang, S., K. Kuroda, F. Le Grand, and M.A. Rudnicki, Asymmetric self-renewal and commitment of satellite stem cells in muscle. Cell, 2007. 129(5): p. 999-1010.

24. Charras, G.T., J.C. Yarrow, M.A. Horton, L. Mahadevan, and T.J. Mitchison, Non-equilibration of hydrostatic pressure in blebbing cells. Nature, 2005. 435(7040): p. 365-9.

25. Charras, G. and E. Paluch, Blebs lead the way: how to migrate without lamellipodia. Nat Rev Mol Cell Biol, 2008. 9(9): p. 730-6.

26. Zammit, P.S., J.P. Golding, Y. Nagata, V. Hudon, T.A. Partridge, and J.R. Beauchamp, Muscle satellite cells adopt divergent fates: a mechanism for self-renewal? J Cell Biol, 2004. 166(3): p. 347-57.

27. Huang, C., Z. Rajfur, C. Borchers, M.D. Schaller, and K. Jacobson, JNK phosphorylates paxillin and regulates cell migration. Nature, 2003. 424(6945): p. 219-23.

28. Han, Z., D.L. Boyle, L. Chang, B. Bennett, M. Karin, L. Yang, A.M. Manning, and G.S. Firestein, cJun $\mathrm{N}$-terminal kinase is required for metalloproteinase expression and joint destruction in inflammatory arthritis. J Clin Invest, 2001. 108(1): p. 73-81.

29. Goddette, D.W. and C. Frieden, Actin polymerization. The mechanism of action of cytochalasin D. J Biol Chem, 1986. 261(34): p. 15974-80.

30. Blaser, H., M. Reichman-Fried, I. Castanon, K. Dumstrei, F.L. Marlow, K. Kawakami, L. SolnicaKrezel, C.P. Heisenberg, and E. Raz, Migration of zebrafish primordial germ cells: a role for myosin contraction and cytoplasmic flow. Dev Cell, 2006. 11(5): p. 613-27.

31. Friedl, P. and K. Wolf, Tumour-cell invasion and migration: diversity and escape mechanisms. Nat Rev Cancer, 2003. 3(5): p. 362-74.

32. Wolf, K., I. Mazo, H. Leung, K. Engelke, U.H. von Andrian, E.I. Deryugina, A.Y. Strongin, E.B. Brocker, and P. Friedl, Compensation mechanism in tumor cell migration: mesenchymalamoeboid transition after blocking of pericellular proteolysis. J Cell Biol, 2003. 160(2): p. 267-77.

33. Frank, S.R., M.R. Adelstein, and S.H. Hansen, GIT2 represses Crk- and Rac1-regulated cell spreading and Cdc42-mediated focal adhesion turnover. EMBO J, 2006. 25(9): p. 1848-59.

34. Shin, I., S. Kim, H. Song, H.R. Kim, and A. Moon, H-Ras-specific activation of Rac-MKK3/6-p38 pathway: its critical role in invasion and migration of breast epithelial cells. J Biol Chem, 2005. 280(15): p. 14675-83.

35. Fackler, O.T. and R. Grosse, Cell motility through plasma membrane blebbing. J Cell Biol, 2008. 181(6): p. 879-84.

36. Pinner, S. and E. Sahai, PDK1 regulates cancer cell motility by antagonising inhibition of ROCK1 by RhoE. Nat Cell Biol, 2008. 10(2): p. 127-37. 
37. Sahai, E. and C.J. Marshall, Differing modes of tumour cell invasion have distinct requirements for Rho/ROCK signalling and extracellular proteolysis. Nat Cell Biol, 2003. 5(8): p. 711-9.

38. Berton, S., B. Belletti, K. Wolf, V. Canzonieri, F. Lovat, A. Vecchione, A. Colombatti, P. Friedl, and G. Baldassarre, The tumor suppressor functions of p27(kip1) include control of the mesenchymal/amoeboid transition. Mol Cell Biol, 2009. 29(18): p. 5031-45.

39. Lee, P.C., M.R. Kibbe, M.J. Schuchert, D.B. Stolz, S.C. Watkins, B.P. Griffith, T.R. Billiar, and L.L. Shears, 2nd, Nitric oxide induces angiogenesis and upregulates alpha(v)beta(3) integrin expression on endothelial cells. Microvasc Res, 2000. 60(3): p. 269-80.

40. Sun, Y., J. Liu, F. Qian, and Q. Xu, Nitric oxide inhibits T cell adhesion and migration by downregulation of beta1-integrin expression in immunologically liver-injured mice. Int Immunopharmacol, 2006. 6(4): p. 616-26.

41. Sanz-Moreno, V., G. Gadea, J. Ahn, H. Paterson, P. Marra, S. Pinner, E. Sahai, and C.J. Marshall, Rac activation and inactivation control plasticity of tumor cell movement. Cell, 2008. 135(3): p. 510-23.

Insert following refs:

Grounds MD and Davies MJ, Chemotaxis in myogenesis Basic and Applied Myology, 1996, 6 (6) p469483.

Gilbert PM, Havenstrite KL, Magnusson KE, Sacco A, Leonardi NA, Kraft P, Nguyen NK, Thrun S, Lutolf MP, Blau HM. Substrate elasticity regulates skeletal muscle stem cell self-renewal in culture. Science. 2010 329(5995):p1078-81.

Griffin CA, Apponi LH, Long KK, Pavlath GK. Chemokine expression and control of muscle cell migration during myogenesis. J Cell Sci. 2010 123(18): p3052-60 


\section{Figure legends}

\section{Figure 1}

Rapid satellite cell migratory phase occurs following emergence from beneath the basal lamina. A. Migration speeds $(\mu \mathrm{m} / \mathrm{h})$ of differing cell types. White bars depict cell lines monitored when cultured on flat in vitro plastic surfaces using lamellipodia-based migration. Black bar shows speed of satellite cells following emergence from the basal lamina on the surface of myofibres using bleb-based cell movement. Significantly higher migration speeds were observed in both MCF10A and satellite cells when compared to $\mathrm{C} 2 \mathrm{C} 12, \mathrm{MDA}-\mathrm{M} 8-231$ and HTR8 cells $(* \mathrm{P}<0.001)$. B. Satellite cell migration velocities following skeletal muscle injury shown in four distinct $18 \mathrm{~h}$ time windows, 0-18, 18-36, 36-54 and 54-72 h post myofibre isolation. Satellite cell migration velocity is significantly increased in the 18-36 and 36-54 h time windows compared to $0-18 \mathrm{~h}(* \mathrm{P}<0.001$ in both cases $)$. Satellite cell migration is significantly slower at the 54-72 $\mathrm{h}$ window compared to $0-18 \mathrm{~h}(* \mathrm{P}<0.005)$. C-K. Immunocytochemical staining of single myofibres using antibodies against Laminin to mark the basal lamina and Pax7 to mark the satellite cell nuclei. C-G. Time course showing a single satellite cell beneath the basal lamina after increasing time points following fibre isolation (A-E, 0, 2, 4, 6 and 12 hrs of culture respectively). Between 4 and 12 hrs post isolation, a pedestal of laminin is built up around the satellite cell (red arrowheads in E-G). H and K. Satellite cells break through the laminin membrane $18 \mathrm{hrs}$ post isolation (red arrowhead). I. Satellite cell localised on the outer surface of the basal lamina (green arrowhead) at $24 \mathrm{~h}$ positioned above a myonucleus that is localised beneath the surface of the lamina membrane (blue arrowhead). J. Dorsal view showing laminin membrane covering the $\operatorname{Pax} 7^{+}$satellite cell 6 hrs following myofibre isolation. Scale bar in $\mathrm{A}=10 \mu \mathrm{m}$.

\section{Figure 2}


Satellite cells display an amoeboid-like shape following emergence from the basal lamina. A-D. SEM images of satellite cells following 18, 24, 48 and $72 \mathrm{hrs}$ of culture respectively. A. SEM image showing a satellite cell emerging through the basal lamina of the myofibre $18 \mathrm{hrs}$ after isolation. B. Satellite cell displaying polarised blebbing. C. Satellite cell showing extensive membrane blebs. D. Cluster of satellite cell progeny all displaying membrane blebs. E. SEM image of a cluster of satellite cells at $72 \mathrm{hrs}$ following treatment with Z-VAD-FMK. Satellite cells continue to show membrane blebbing. Scale bar in $\mathrm{A}=2 \mu \mathrm{m}$. Scale bar in $\mathrm{B}=5 \mu \mathrm{m}$. $\mathrm{B}$ and $\mathrm{C}$ are taken at the same magnification. Scale bar in $\mathrm{D}$ and $\mathrm{E}=10$ $\mu \mathrm{m}$.

\section{Figure 3}

iNOS and eNOS are expressed during the satellite cell migratory phases. A. Immunocytochemical staining showing the expression of iNOS and Pax7 in a cluster of satellite cell progeny following $72 \mathrm{~h}$ of culture. Expression of iNOS is localised to the nucleus of both Pax $7^{+}$(yellow arrowheads) and $\mathrm{Pax}^{-}$(red arrowheads) satellite cells. B. Immunocytochemical staining showing the expression of eNOS and Pax7 in a single satellite cell (top row), a satellite cell doublet (middle row) and a small cluster of progeny cells (bottom row) following $48 \mathrm{~h}$ of culture. eNOS expression is varied in its localisation. Some satellite cells display uniform cytoplasmic expression (top row). Some cells show polarised expression (middle and bottom rows-red arrowheads) and some cells show low levels of expression (bottom row-blue arrowhead). Scale bar in $\mathrm{A}$ and $\mathrm{B}=15$ and $10 \mu \mathrm{m}$ respectively.

\section{Figure 4}

$N O$ and PCP pathways play key roles in satellite cell migration. A. Average satellite cell migration velocities during $0-18,18-36$ and $36-54 \mathrm{~h}$ time windows of culture following treatment with PCP inhibitor SP600125 or the NOS inhibitor L-NAME. L-NAME significantly reduced satellite cell migration between $0-18,18-36$ and $36-54 \mathrm{~h}\left({ }^{*} \mathrm{P}<0.001\right.$ at all time points respectively). SP600125 caused 
significant reductions in migration velocity between $18-36$ and $36-54 \mathrm{~h}$ time windows only $(* \mathrm{P}<0.001$ and * $\mathrm{P}=0.002$ respectively). B. Average linear movement of all satellite cells measured throughout the entire time course of study (0-72 h of culture). Linearity of movement was calculated as the final distance from the quiescent niche of the progeny cells divided by the total distance migrated. SP600125 and L-NAME had no significant effect on migration linearity of satellite cells.

\section{Figure 5}

Reduced satellite cell migration velocity induced by SP600125 or L-Name is accompanied by a reduction in bleb number. A. Average number of blebs per satellite cell quantified from SEM images of satellite cells following culture in control conditions or in the presence of $25 \mu \mathrm{M}$ SP600125 or $5 \mu \mathrm{M}$ L-NAME. Control satellite cells displayed increased numbers of blebs over time, with significantly more blebs present on cells at $72 \mathrm{~h}$ compared to $24\left({ }^{+} \mathrm{P}=0.027\right)$. At $24 \mathrm{~h}$ post-isolation, fewer blebs were noted on both SP600125- and L-NAME-treated satellite cells. At $48 \mathrm{~h}$ post-isolation, SP600125- and L-NAMEtreated satellite cells both displayed significantly fewer blebs than control cells $\left({ }^{*} \mathrm{P}=0.05\right.$ and $* \mathrm{P}=0.017$ respectively). At $72 \mathrm{~h}$ post-isolation, SP600125- and L-NAME-treated satellite cells both displayed significantly fewer blebs than control cells $\left({ }^{*} \mathrm{P}<0.001\right.$ in both cases). B. Average velocity of bleb initiation and retraction on control, SP600125- and L-NAME-treated satellite cells. No significant differences in bleb dynamics were observed. C. SEM images showing satellite cells on control, SP600125- and L-NAME-treated myofibres following 24, 48 and $72 \mathrm{~h}$ of culture. D. Average percentage of satellite cell progeny positive for Pax7 and/or Myogenin following $72 \mathrm{~h}$ of single myofibre culture. A significantly greater number of $\mathrm{Pax}^{+}$cells were observed in L-NAME treated cultures compared to control (*P=0.001). Significantly fewer myogenin+ cells were observed in both SP600125- and LNAME-treated cultures ( $* \mathrm{P}=0.006$ and $* \mathrm{P}<0.001$ respectively). Scale bar in $\mathrm{C}=5 \mu \mathrm{m}$, all panels are at the same magnification. 


\section{Figure 6}

Actin polymerisation is required for the maintenance of bleb formation on satellite cells. A-C. High power phase contrast microscopy images of an individual satellite cell following 48 hrs of culture. A. Satellite cell in control conditions. Black arrowheads mark small distinct membrane blebs. B-C. Image of the same satellite cell shown in A after treatment with $1 \mu \mathrm{M}$ Cytochalasin-D. B Satellite cell shown in A 80 seconds post-treatment with Cytochalasin-D; large blebs form in the cell membrane (white arrowheads). C Satellite cell shown in A and B, 9 minutes following treatment; the same large blebs shown in B have not been retracted back into the cell (white arrowheads) and no new blebs have formed. D-I. SEM images of satellite cells on isolated single myofibres following culture in control conditions (DF) or treated with Y-27632 (G-I). Y-27632 was added to treated cultures at $0(\mathrm{G}), 24(\mathrm{H})$ and 48 hours (I) post isolation. Control cells display a blebbing morphology however Y-27632 treated cells form no blebs and display elongated lamellipodia. Scale bar in $\mathrm{D}-\mathrm{F}=5 \mu \mathrm{m}$, and in G-I=5 $\mu \mathrm{m}$.

\section{Supplementary Figure 1}

Satellite cells are highly motile following activation and proliferate outside the basal lamina.

A-F. Individual frames from supplementary movie 1 taken using phase contrast microscopy. A. Single satellite cell on the surface of the myofibre $32 \mathrm{~h} 45^{\prime}$ following isolation (yellow arrowhead). B. The same cell shown in A immediately prior to the initial mitotic event after $36 \mathrm{~h} 45^{\prime}$ of culture (yellow arrowhead). C. Initial satellite cell mitotic event on the outer surface of the basal lamina $37 \mathrm{~h} 30^{\prime}$ after isolation, yellow arrowheads indicate two progeny cells, red line depicts to plane of division - perpendicular to the myofibre. D. Yellow arrowheads mark the two progeny cells of the mitotic event shown in C. After $39 \mathrm{~h}$ 15 ' of culture, the cells have migrated away from the initial site of division in opposing directions. E. Yellow arrowheads indicate progeny cells on the surface of the myofibre following single mitotic events in both of the cells shown in D after $52 \mathrm{~h} 15^{\prime}$ of culture. F. Clusters of progeny formed after $72 \mathrm{~h} 15^{\prime}$ from 
the original two cells shown in D are highlighted (yellow arrowheads). G. SEM image displaying the satellite cell progeny immediately after the initial mitotic event, perpendicular to the plane of the myofibre, $48 \mathrm{~h}$ after myofibre isolation. H. Schematic depicting the initial migratory events of the satellite cell during activation and initial division. Key: Green ovals-satellite cell, red line-basal lamina, black arrow-slow movement, green arrow-accelerated movement, red arrow-fast movement, blue arrowdirection of division. I-K. Individual phase contrast time lapse movie frames showing a cluster of satellite cell progeny migrating as a cohort of cells along the myofibre (I-J yellow arrowheads) and subsequently splitting into two separate groups of cells (K-yellow arrowheads). Scale bar in A, I, L and $\mathrm{O}=40 \mu \mathrm{m}, \mathrm{A}-\mathrm{F}$, I-K, L-N and O-Q are taken at the same magnification respectively. Scale bar in G=10 $\mu \mathrm{m}$.

\section{Supplementary Figure 2}

NO and PCP signalling regulate C2C12 myoblast cell migration through separate mechanisms. Average velocity of confluent C2C12 cells cultured in either control, L-NAME, SP600125 or SP600125+LNAME treated conditions. Significantly lower migration velocities were observed in all treated cultures when compared to control conditions $(* \mathrm{P}=0.029, * \mathrm{P}<0.001$ and $* \mathrm{P}<0.001$ respectively). SP600125 treated cultures displayed significantly less migration than L-NAME treated cells $(\mathrm{P}<0.001)$. SP600125+LNAME treated cultures showed significantly less movement than L-NAME and SP600125 treated cultures $(\mathrm{P}<0.001$ and $\mathrm{P}=0.018$ respectively).

\section{Supplementary movie 1}

